Slavica

bruxellensia

\section{Slavica bruxellensia}

Revue polyphonique de littérature, culture et histoire

slaves

5 | 2010

Après 1989

\title{
Les littératures polonaise et biélorusse dans le contexte du postmodernisme
}

\section{Natalia Dzienisiuk}

Traducteur : Katia Vandenborre

\section{(2) OpenEdition}

\section{Journals}

Édition électronique

URL : http://journals.openedition.org/slavica/299

DOI : 10.4000/slavica.299

ISSN : 2034-6395

\section{Éditeur}

Université libre de Bruxelles - ULB

Édition imprimée

Pagination : 34-48

ISSN : 2031-7654

\section{Référence électronique}

Natalia Dzienisiuk, «Les littératures polonaise et biélorusse dans le contexte du postmodernisme »,

Slavica bruxellensia [En ligne], 5 | 2010, mis en ligne le 15 février 2010, consulté le 19 avril 2019. URL: http://journals.openedition.org/slavica/299; DOl : 10.4000/slavica.299

\section{Ce document a été généré automatiquement le 19 avril 2019.}

\section{(c) (i) (9)}

Les contenus de Slavica bruxellensia sont mis à disposition selon les termes de la Licence Creative Commons Attribution - Pas d'Utilisation Commerciale - Pas de Modification 3.0 France. 


\title{
Les littératures polonaise et biélorusse dans le contexte du postmodernisme
}

\author{
Natalia Dzienisiuk \\ Traduction : Katia Vandenborre
}

\section{NOTE DE L'ÉDITEUR}

Cet article a initialement été écrit en polonais

1 Il existe de multiples manières philosophiques et artistiques d'essayer de comprendre le monde. Le postmodernisme en est une parmi d'autres. Ce modèle de pensée décrit la réalité non pas par le biais d'un système de concepts ou d'images, mais à l'aide de moyens linguistiques réalisés dans le texte. Les postmodernistes perçoivent le monde comme un espace textuel mouvant et décentralisé qui comprend plusieurs niveaux. Celui-ci s'enrichit sans cesse de nouveaux textes par la transformation et l'arrangement de textes déjà existants. Le texte est déterminé par la conscience de l'homme qui perçoit non pas la réalité environnante mais un ensemble de signes qu'il déchiffre en fonction de la situation culturelle, historique et nationale. L'esthétique du postmodernisme se base sur le dialogue culturel. Dans les œuvres postmodernistes, des figures historiques de différentes époques s'efforcent de se comprendre, l'action se développe dans un cadre qui réunit de manière originale des composantes propres à différentes traditions socioculturelles ou nationales, enfin on assiste à un renouvellement de l'atmosphère de polyphonie culturelle et de manque de détermination temporelle. Le passé devient l'objet de la déconstruction postmoderniste, ce qui permet de regarder l'histoire contemporaine à travers le prisme historique, mais aussi, à l'inverse, de juger ce qui a eu lieu dans l'histoire d'un point de vue actuel. La tradition culturelle de chaque peuple s'inscrit de façon organique dans le contexte de la culture mondiale, elle l'élargit, en modifie le 
contenu et, en même temps, elle y acquiert une nouvelle interprétation en rapport avec les autres traditions, révélant ainsi une intertextualité entre les cultures.

Dans le présent article, j'analyserai dans les littératures polonaise et biélorusse les problèmes fondamentaux d'histoire littéraire, de philosophie et d'esthétique liés à la naissance du postmodernisme. J'étudierai les tendances générales de formation de ce courant dans le cadre de la nouvelle situation culturelle apparue dans les deux pays après 1989, ce qui mettra en évidence des techniques et des stratégies postmodernistes communes aux écrivains polonais et biélorusses. La confrontation des processus littéraires modernes révèlera les particularités typologiques et les traits régionaux des postmodernismes polonais et biélorusse dans un contexte mondial.

\section{Le postmodernisme...}

3 Le postmodernisme comme système particulier de conceptions artistique, esthétique et scientifique du monde est apparu dans la critique, littéraire, scientifique et philosophique américaine dans la deuxième moitié $\mathrm{du} \mathrm{XX} \mathrm{X}^{\mathrm{e}}$ siècle. Il était le résultat de la réflexion sur les événements tragiques de l'histoire mondiale récente, conséquence du désenchantement quant aux possibilités de connaissance rationnelle et d'ordonnancement de la réalité, mais aussi une tentative de réévaluer la modernité passée avec son culte du beau, de l'intellect et de l'aspiration à l'idéal. Dans les nouvelles conditions historico-sociologiques d'existence de la civilisation, les normes morales et les priorités axiologiques sont mises en doute, et «l'éthique s'est transformée en concept périphérique »" , ne pouvant plus de ce fait être traitée de façon objective.

4 En Amérique, où «le mélange de cultures, d'attitudes morales, de mœurs, de races, de religions était et est le plus grand, cela a aussi mené le plus souvent à la cristallisation d'une nouvelle sensibilité et d'une nouvelle conscience $»^{2}$, laquelle s'est progressivement répandue dans les pays d'Europe de l'ouest. Dans les années 1970 et au début des années 1980, les conceptions postmodernistes sont devenues l'objet de la pensée théorique critique et de la pratique artistique européenne, principalement française, italienne, et ensuite allemande et anglaise. Les idées de penseurs tels que Jacques Derrida, JeanFrançois Lyotard, Gilles Deleuze, Félix Guattari, Richard Rorty, Michel Foucault, Roland Barthes, Julia Kristeva et d'autres ont joué un rôle important dans le développement, la systématisation et la validation de la théorie postmoderniste. C'est justement dans leurs travaux que sont apparues pour la première fois les thèses qui deviendront plus tard les slogans les plus populaires du postmodernisme: «le monde comme un chaos», «le décentrement», "la déconstruction de l'ordre hiérarchique des éléments», «la perception du monde comme un texte », «l'intertexte », «l'intertextualité », «la pensée poétique », «la mort du sujet (de l'auteur)», «l'histoire comme volonté du hasard aveugle », « la limite des grandes narrations », etc.

5 La formation du postmodernisme dans l'art d'Europe occidentale vient de l'épuisement des conventions jusque là dominantes dans la littérature et de l'affaiblissement des positions esthétiques du modernisme incapable de trouver l'harmonie dans la réalité chaotique. Si la littérature moderniste était condamnée à l'élitisme et à l'hermétisme (mentionnons ici les œuvres de James Joyce, T. S. Eliot, Thomas Mann, Robert Musil, etc.), le postmodernisme recherche des moyens de sortir des salons artistiques et des bibliothèques académiques. En mettant en question la hiérarchie traditionnelle des 
genres, les postmodernistes peuvent s'approprier les différentes formes de la littérature populaire. Cette dernière attire les écrivains par le caractère de sa narration, par son pouvoir de jouer avec le lecteur, jeu qui se caractérise par des « règles indéterminées qui changent continuellement $\rrbracket^{3}$. Les œuvres des postmodernistes ne se limitent pas à la poétique des genres populaires. Ils en font plutôt une parodie raffinée. Les écrivains postmodernistes ne prétendent pas inventer une nouvelle façon absolue de représenter la réalité. Au contraire, ils se tournent vers des moyens artistiques et des méthodes narratives déjà existants. De là résulte un pluralisme stylistique qui repose sur la coexistence et la synergie de différents styles artistiques et de conventions narratives. Le pluralisme des regards, la multiplication des sens, l'intertextualité, le scepticisme quant à la connaissance de la réalité marquent la conscience de l'homme contemporain.

\section{... en Pologne ...}

6 Comme le postmodernisme est reconnu comme «une culture pluraliste, mélangée, polyphonique, populaire, réunissant différents niveaux et différentes langues, sensible au manque de naturel, à la théâtralité, au jeu avec les conventions, se servant volontiers de la mystification, de l'ironie et de la parodie $»^{4}$, l'acceptation de l'esthétique postmoderniste devient possible dans des pays ouverts au dialogue interculturel, économique et politique, dans des états respectant la liberté de parole, la diversité des visions du monde et le droit des minorités ethniques, culturelles, sexuelles, etc., dans les cultures libres de tout dictat politique, idéologique ou esthétique. C'est pourquoi, dans les pays de l'ex-bloc socialiste, le postmodernisme a commencé à être connu, reconnu et reproduit seulement après la chute du système totalitaire communiste au tournant des années 1980 et 1990. Pendant près de cinquante ans (et dans le cas de l'URSS pendant plus de septante ans), la littérature et l'art en Europe centrale et orientale se sont développés dans l'isolement des processus en œuvre dans la culture occidentale, dont les sociétés reposaient sur les principes de démocratie et de liberté créatrice. Ils restaient soumis aux impératifs normatifs et à la pression politique qui faisaient en sorte de contrôler et d'assurer une production artistique conforme à l'orientation donnée. Cependant, même quand l'enthousiasme pour "les perspectives lumineuses de la construction socialiste » était le plus grand, il y avait des écrivains qui, comprenant l'anormalité des conditions imposée à la littérature, cherchaient leurs propres manières de vaincre les canons totalitaires et créaient des modèles artistiques alternatifs, et ce à l'encontre des impératifs dominants du réalisme socialiste. Ces tendances avaient une origine double: elles venaient tant de l'intérieur, où elles étaient dues à la pression des forces révisionnistes, que de l'extérieur, étant le produit de diverses inspirations occidentales, en ce compris d'émigration. Déjà dans les circonstances de $1956^{5}$, la culture polonaise a rejeté le caractère totalitaire, c'est-à-dire uniforme et imposé, de l'art réaliste socialiste et s'est largement ouverte à la diversité des langues et des philosophies. Cela a été favorisé par divers facteurs : le dégel temporaire dans les relations polono-soviétiques, l'obtention par la Pologne d'une plus grande liberté dans la politique intérieure, l'arrivée au pouvoir de Władysław Gomułka qui s'efforçait alors de réaliser des réformes visant la libéralisation du régime étatique et le renforcement du statut indépendant de la Pologne dans le monde.

7 Après 1956, la vie culturelle du pays se mobilisa. Ce fut justement à ce moment, après qu'on eût mis fin à la censure dans l'art et l'édition, que fut réhabilité, réédité et 
décemment analysé un ensemble de textes avant-gardistes de Stanisław Ignacy Witkiewicz (Witkacy), Witold Gombrowicz, Bruno Schulz ou encore Michał Choromański. Une nouvelle génération d'écrivains vit le jour, parmi lesquels la riche pléiade de poètes connus comme étant la génération de «Współczesność » (Contemporainéité), du nom de la revue qui les publiait. Ajoutons le développement spontané de cabarets étudiants (le Bim-Bom à Gdańsk et Studencki Teatr Satyryków [Théâtre Étudiant des Satiristes, STS] à Varsovie voient déjà le jour en 1954) et de clubs de discussion de différents genres (le Klub Krzywego Koła [Club du Cercle Tordu] était actif à Varsovie dès le 5 janvier 1956), lesquels concentraient l'élite de l'intelligentsia polonaise de l'époque. Mais également des cafés littéraires (apparus auprès de maisons d'édition telles que Państwowy Instytut Wydawniczy, «Czytelnik », Wydawnictwo Literackie), dont le " Piwnica pod Baranami » avec Piot Skrzynecki et le théâtre "Cricot II » de Tadeusz Kantor à Cracovie, le «Studio Pantomimy " (Studio de la Pantomime) de Henryk Tomaszewski à Wrocław. En 1959 commence l'activité du fameux « Teatr 13 RzĘdów » (Théâtre des 13 Rangs) à Opole sous la direction de Jerzy Grotowski et Ludwik Flaszen.

Un groupe composé des « fils spirituels » de Witkacy et de Gombrowicz se distingua parmi les jeunes créateurs : Sławomir Mrożek, Ireneusz Iredyński, Andrzej Kuśniewicz (bien que du même âge que ceux-ci, il débuta beaucoup plus tard), Stanisław Zieliński (un peu plus jeune), Piotr Wojciechowski, Wilhelm Mach. Le courant absurde et grotesque dans le théâtre polonais, dont on trouve les fondements dans le grotesque macabre des pièces de Witkacy, devint progressivement un phénomène à part. Tadeusz Różewicz, Mrożek, Iredyński, Gombrowicz (en émigration) donnèrent un tour particulier à ce type d'écriture. Cette époque connut aussi le riche développement de l'œuvre de Stanisław Lem, dont les romans de science-fiction attirèrent des millions de lecteurs en Pologne et dans le monde.

Pendant cette période, commencèrent à paraître des revues qui furent d'une importance capitale pour la culture et la conscience polonaises : «WSPÓECZESNOŚć », « DIALOG », «WIĘŹ ", « ODRA », « PRZEGLĄD HUMANISTYCZNY », et l'édition renouvelée du « ROCZNIK LITERACKI ». Des écrivains importants pour la littérature polonaise revinrent d'émigration: Choromański, Melchior Wańkowicz, Antoni Słonimski, Maria Kuncewiczowa, Stanisław Cat-Mackiewicz, Teodor Parnicki. Les publications des écrivains émigrés (par exemple Gombrowicz) commencèrent aussi à paraître, bien que leur nombre fût relativement réduit.

L'éducation culturelle des Polonais s'accéléra brusquement. La conscience polonaise s'appropria activement les œuvres artistiques et philosophiques occidentales. Elle était intellectuellement prête à recevoir une nouvelle langue, un nouveau style artistique.

11 En considérant «le recul par rapport à un modèle canonique d'art, l'acceptation de plusieurs langues ou codes, le rejet de toute forme de totalitarisme et d'autoritarisme ${ }^{6}$ comme les principaux signes de la culture postmoderniste, il est sans aucun doute possible d'affirmer qu'un tel changement s'est accompli après 1956.

Toutefois, la question de la détermination d'une limite inférieure au postmodernisme littéraire en Pologne, et donc des textes et des auteurs reconnus comme postmodernistes, reste contestable. Ewa Wiegandt, par exemple, suggère que :

Edward Możejko considère Góry nad czarnym morzem (Les montagnes sur la mer noire) (1961) de Mach ainsi que la prose de science-fiction de Lem, et tout particulièrement Kongres futurologiczny (Le congrès de futurologie) (1973), comme la limite inférieure de la 
littérature postmoderniste ${ }^{8}$. Le critique situe l'étape suivante de l'évolution de la littérature postmoderniste au niveau des œuvres des écrivains réunis autour de la revue " TWóRczość », avec l'exemple extrême du roman de Krzysztof Bielecki End \& Fin Company, publié dans la deuxième moitié des années 1980. Par contre, Krzysztof Uniłowski reconnaît les présentations publiées dans les colonnes de "TWóRczość » comme les premières du genre postmoderniste :

La difficulté de définir l'étendue de la littérature postmoderniste en Pologne tient, d'une part, à la complexité et à l'ambigüité du terme " postmodernisme » même, qui, sans être en soi tout à fait défini, contient par nature une multiplicité de significations (il n'est pas tout à fait définissable notamment parce qu'il implique une réflexion sur des phénomènes relativement nouveaux voire très récents). Elle est, d'autre part, liée à la conviction très répandue qu'on ne peut pas parler de postmodernisme dans la littérature polonaise tant que ce terme n'est pas en vigueur et qu'il n'a pas fait l'objet d'études en polonistique.

Le terme "postmodernisme » est entré dans le vocabulaire de la critique polonaise en 1983 lors de l'édition de l'anthologie de manifestes et d'essais d'écrivains américains Nowa proza amerykańska ${ }^{10}$ (Nouvelle prose américaine). Pendant plusieurs années, il fut utilisé exclusivement pour désigner les phénomènes les plus récents en littérature étrangère. Les tentatives de le rapporter à la sphère polonaise ont suscité de vives discussions au début des années 1990, en raison de la nomenclature lexicale nationale traditionnelle. Les revues littéraires « DIALOG », « LITERATURA NA ŚWIECIE », « TEKSTY DRUGIE » et le trimestriel « FA-ART » publié par l'Université de Silésie ont joué un rôle clé dans la diffusion des idées postmodernistes, en ce compris l'interprétation postmoderniste de la littérature polonaise contemporaine. Un numéro spécial de « TEKSTY DRUGIE » (1993/1) consacré à la problématique postmoderniste présentait les opinions divergentes de célèbres critiques et chercheurs sur le phénomène du postmodernisme dans la littérature polonaise : tant des opinions négatives (Włodzimierz Bolecki ${ }^{11}$ ) que positives (Zdzisław Łapiński ${ }^{12}$ ), ou sceptiques (Kazimierz Bartoszyński ${ }^{13}$ ).

16 À mon avis, on ne peut concilier les différentes hypothèses sur la nouvelle situation littéraire en Pologne que si l'on tient compte du fait que le postmodernisme dans la littérature polonaise (comme dans d'autres pays d'Europe centrale et orientale) peut être appréhendé dans deux sens au moins. Dans le sens le plus général, le postmodernisme désigne un type particulier de pensée artistique qui lie de manière organique une autoréflexion philosophique, une perception des réalités de la vie par l'intuition ou par associations, une connaissance méditative du monde par l'actualisation des signes de l'inconscient, la mémoire génétique et collective, l'imitation de l'héritage culturel et de l'expérience historique, etc. Si l'on comprend le concept ainsi, le postmodernisme est repérable dans la littérature à partir de la fin des années 1950. Par contre, le deuxième sens du mot, c'est-à-dire un courant littéraire défini, ne peut être rapporté qu'aux créations artistiques produites à la charnière des années 1980 et 1990.

Les critiques littéraires polonais classent le plus souvent dans le courant postmoderniste : Mury Hebronu (Les murs d'Hébron, 1992) et Biały kruk (Le Corbeau blanc, 1994) d'Andrzej Stasiuk; Rien ne va plus (1992) d'Andrzej Bart; Spis cudzolożnic (La Liste des femmes adultères, 1993) de Jerzy Pilch; Panna Nikt (Mademoiselle Personne, 1993) de Tomek Tryzna ; les romans de Manuela Gretkowska (1991-1998) ; Podróż ludzi KsiĘgi (Le Voyage des gens du Livre, 1993), E.E. (1995) et Dom dzienny, dom nocny (Maison de jour, maison de nuit, 1998) d'Olga Tokarczuk ; Absolutna amnezja (L'Amnésie absolue, 1995), les nouvelles des livres Śmierć $i$ spirala (La Mort et la spirale, 1992) et Niebieska menażeria (La Ménagerie 
bleue, 1997) d'Izabela Filipiak; Biały kamień (La Pierre blanche, 1994) et Kochany Franz (Mon cher Franz, 1999) d'Anna Bolecka ; Zapiski z nocnych dyżurów (Notes des permanences de nuit, 1995) de Jacek Baczak ; Sny i kamienie (Rêves et pierres, 1995) et $W$ czerwieni (Dans le rouge, 1998) de Magdalena Tulli; Tabu (Tabou, 1998) et Obciach (Blâmage, 1999) de Kinga Dunin ; la microfiction de Natasza Goerke (1994-1999) ; les nouvelles de Krzysztof Varga (1993-1998) ; les récits de Paweł Huelle, etc.

Les écrivains polonais contemporains se servent de différentes techniques et stratégies postmodernistes : 1) ils accentuent la multiplicité des sens, la polyphonie de la réalité à l'aide d'une langue actualisée à différents niveaux de son fonctionnement, dans différents discours (esthétique, philosophique, scientifique, culturel, social, politique, etc.) ; 2) en établissant un dialogue entre les conventions de l'époque et les œuvres littéraires, ils entreprennent un jeu intertextuel à l'aide de citations, allusions, parodies, pastiches, collages ; 3) en réfutant les stéréotypes, les mythes, les convictions collectives de la société, et ce en les transformant de façon ironique dans le texte; 4) ils rejettent les grandes narrations, ils créent une image de la vie comme celle d'une aventure privée. D'une part, les écrivains polonais renoncent aux possibilités mimétiques et narratives de la création de texte, ils rejettent la linéarité du récit, ils accordent une grande importance à la fiction comme moyen de connaître et de surmonter la réalité, ils révèlent le morcellement de la pensée postmoderne, le caractère fragmentaire du temps, de l'espace, de l'information par une structure digressive de la narration et l'introduction de trames parallèles, ils se réfèrent à l'auto-thématisme et à l'autoréflexion. Mais d'un autre côté, les postmodernistes continuent d'utiliser la convention de fabulation, ils se servent de la tradition de façon consumériste, appliquant dans les œuvres le double code de l'art postmoderniste.

19 L'analyse des textes littéraires et critiques de ces dernières décennies montre la pénétration des conceptions philosophiques et esthétiques postmodernistes dans la culture polonaise. Elle montre que le canon littéraire reconnu sans conteste comme postmoderniste s'est élargi à des œuvres nouvelles qui se servent des stratégies littéraires du postmodernisme.

\section{... et en Biélorussie}

En Biélorussie et dans les autres pays de l'espace postsoviétique, le changement historicoculturel s'est produit à la fin des années 1980 et au début des années 1990. Il a été conditionné par un ensemble de facteurs sociopolitiques, socio-psychologiques, économiques et culturels interconnectés. La chute de l'état hégémonique centralisé de type autoritaire, le recouvrement de l'indépendance par les anciens pays soviétiques et la possibilité d'introduire sa propre politique nationale ont entraîné un réexamen des valeurs humaines et sociales (la démocratie, la liberté, l'indépendance, l'activité sociale sont devenues les plus importantes) et ont réveillé la dignité nationale dans ces pays qui n'avaient pas le droit à l'autodétermination et à un plein développement de leur propre culture pendant plusieurs décennies car ils étaient les otages du régime communiste. La chute du « rideau de fer » a favorisé l'activation des contacts culturels entre la Biélorussie et les pays d'Europe occidentale et les USA. La nouvelle réalité a entraîné la formation d'une nouvelle pensée qui n'était toutefois pas tout à fait dépouillée de stéréotypes du passé. 
21 Au tournant des années 1980 et 1990, l'art biélorusse a été fortement stimulé. Une nouvelle génération d'écrivains a vu le jour. Les changements politiques, la démocratisation progressive de la vie sociale ont rendu possible la liberté de création, dégagée de toute orientation idéologique, du schématisme de la narration et de la limitation de la pensée de l'écrivain par les principes du réalisme socialiste. Dans la vague de renaissance nationale, les œuvres des jeunes écrivains étaient imprégnées d'optimisme, du pathos de la victoire contre le récent passé socialiste, elles lançaient un défi courageux à la population passive, au système politique, au monde entier, étant pénétrées d'une foi inébranlable dans les changements positifs qui se produisaient dans la société biélorusse.

Le mouvement littéraire s'activa à cette période. De nouvelles associations littéraires virent le jour («Tutejšyja », Tavapystva VoL̀nykh Litaratarau [TVL, Association des Écrivains Libres], Bum-Bam-Lit [BBL]), de nombreux livres biélorusses furent édités et réédités, de nouvelles revues littéraires, des journaux, des volumes amateurs et d'autres maisons d'édition apparurent (les almanachs «BLAKITNY LIKHTAR ", " MILAVICA », « LITARATURA », les almanachs de TVL « KALOS'SE », « NAŠA NIVA », « KSERAKS BELARUSKI »). Le spectre générique des œuvres biélorusses s'élargit, les thématiques se diversifièrent. La nouvelle réalité imposait de rechercher de nouveaux moyens linguistiques et stylistiques, de nouvelles façons de la refléter. Le type d'écriture changea progressivement. Les écrivains contemporains s'écartèrent de plus en plus souvent de la métanarration, c'està-dire des "grandes histoires ", préférant des petites histoires fragmentaires de la vie privée, ils renonçaient aux manières traditionnelles de refléter la réalité et se tournaient consciemment ou intuitivement dans leurs œuvres vers des procédés littéraires, des moyens artistiques et des figures de style propres à la poétique du postmodernisme.

Ainsi, dans la première moitié des années 1990, s’est formée en Biélorussie une « nouvelle situation littéraire ", terme introduit par le critique, poète, prosateur et essayiste Sjargej Dubavec en vue d'universaliser et de définir les nouveaux phénomènes dans la littérature biélorusse à la fin du $\mathrm{XX}^{\mathrm{e}}$ siècle. Il se caractérise par une diversité de formes stylistiques, un pluralisme des méthodes artistiques, des courants, des orientations, des conceptions philosophiques et esthétiques, la liberté de créer, des audacieuses expérimentations linguistiques, parfois agressivement étourdissantes, d'actives recherches de différentes manières d'autoréflexion, etc. Le discours postmoderniste s'affermit ainsi dans la littérature biélorusse.

24 La naissance du phénomène culturel nouveau que fut le postmodernisme était conditionnée par des événements sociopolitiques, la formation d'une nouvelle conscience sur les ruines de la pensée stéréotypée de l'homme soviétique, la fin de l'isolement culturel du pays, mais aussi la crise de l'esthétique réaliste socialiste. L'art qui assouvissait les besoins idéologiques et esthétiques du citoyen soviétique dans les années 1980 sembla inadapté à la nouvelle situation socioculturelle. Le vide en matière d'art alternatif fut rempli par le postmodernisme, alors déjà célèbre en Europe, car celui-ci traduisait le mieux le sentiment de crise de l'époque précédente et de manque de confiance dans les temps modernes, d'instabilité et de confusion du monde.

Le terme "postmodernisme " apparaît pour la première fois dans la critique littéraire biélorusse en 1994. Quelques essais consacrés au postmodernisme dans la philosophie et dans l'art aux États-Unis et en Europe occidentale parurent dans les colonnes du cahier littéraire et philosophique «ZNO ", lequel était édité par un groupe d'intellectuels 
biélorusses entre 1993 et 1997 comme supplément mensuel du journal « KUL'TURA ». Les auteurs biélorusses réfléchirent sur le problème de la réception des conceptions postmodernistes dans le but d'ouvrir de nouveaux horizons dans la littérature biélorusse. Cependant, les études littéraires académiques étaient dominées par une perception sceptique voire négative de cette tendance de tradition étrangère, les textes contemporains étant analysés au moyen d'une méthodologie positiviste. Par contre, les philosophes, les spécialistes en culture, en esthétique, en littérature russe ou étrangère ont beaucoup fait pour populariser et développer un appareil conceptuel postmoderniste. Il convient de citer les travaux scientifiques de Maryna Mažejka, Aljaksandr Grycanau, Tamara Tuzava, AL'mira Usmanava, les livres de Iryna Skarapanova Russkaja postmodernistskaja literatura (La littérature postmoderniste russe) et Russkaja postmodernistskaja literatura: novaja filosofija, novyj jazyk (La littérature postmoderniste russe: nouvelle philosophie, nouvelle langue), mais aussi l'encyclopédie du postmodernisme ${ }^{14}$, qui constitue l'étude la plus approfondie de l'espace littéraire postsoviétique.

La connaissance des conceptions esthétiques et philosophiques du postmodernisme et des modèles étrangers de la littérature postmoderniste a poussé à rechercher un sociocode postmoderniste tant dans les textes les plus récents des écrivains de la jeune génération que dans des œuvres plus anciennes de la littérature biélorusse d'après 1960. Certaines stratégies postmodernistes étaient appliquées, consciemment ou intuitivement, mais indépendamment de ce courant littéraire, par des créateurs des associations littéraires « Tutejšyja » et TVL, comme par exemple Dubavec, Adam Globus, Igar Babkou, Maksim Klimkovič, Ales' Arkuš, Jury Gumjanjuk, Igar Sidaruk, Ljavon VoL'ski et d'autres déjà dans les années 1980. Nous trouvons encore avant certains traits du postmodernisme dans l'œuvre du classique de la littérature biélorusse Uladzimir Karatkevič, tout particulièrement dans son roman Khrystos pryzjamliusja u Garodni (Le Christ a atterri à Grodno), lequel a paru à la moitié des années 1960, quelques années avant le roman d'Umberto Eco Le nom de la rose, qui est considéré actuellement comme un modèle de littérature postmoderniste.

L'examen approfondi de l'œuvre de Karatkevič et l'analyse de la singularité de ses œuvres artistiques en rapport avec la nouvelle situation culturelle en Europe et en Biélorussie ont permis aux chercheurs de distinguer certaines stratégies du postmodernisme littéraire dans le roman Khrystos pryzjamliusja u Garodni. Autrement dit, l'écrivain applique le principe du collage : le matériel servant à produire le texte de l'œuvre est constitué non seulement de la pensée de l'auteur, des idées et des moyens de les réaliser, mais aussi de textes littéraires connus, assimilés sur le plan culturel, ou certaines de leurs parties. Pour Karatkevič, les histoires de l'Évangile, les fragments de chroniques de la Rus ou de l'ancienne Lituanie (par exemple les chroniques de Maciej Stryjkowski ${ }^{15}$ ), les œuvres historiographiques, les fragments d'œuvres littéraires, les documents historiques (les poèmes d'Hésiode, DanteAlighieri, Johann Wolfgang von Goethe, Rudyard Kipling, Conrad Meyer, Giordano Bruno, Francysk Skaryna, François Rabelais, Catulle), l'apocryphe biélorusse médiéval, les recommandations aux acteurs, les ballades, les épigrammes en latin, le code d'honneur des chevaliers, les sermons, les légendes, les légendes de saints, les chants gaéliques, biélorusses, les proverbes, les fables, les hymnes, les sagas, etc. sont autant de sources textuelles ${ }^{16}$.

Dans les romans de Karatkevič, la conception caractéristique du postmodernisme qu'est la carnavalisation du monde est appliquée dans tout l'espace de l'œuvre. Dès le début, les 
héros sont forcés de mettre les masques du Christ "paysan » et des douze apôtres, et de voyager à travers le pays dans un fourgon de théâtre. Néanmoins, alors qu'au début leurs activités sont en accord avec la fable biblique, leurs rôles se mettent ensuite à dicter leurs actes, leur comportement et leur caractère. De là résulte le conditionnement du récit par le texte source (dans ce cas l'Évangile), ce qui est une propriété des textes postmodernistes. Mais dans le roman, ce conditionnement n'est que partiellement réalisé. Le plus important reste l'idée de l'auteur qui se base sur la fable biblique, qu'il introduit toutefois à un nouveau niveau.

De nombreux personnages et situations dans le roman peuvent être perçus de différentes manières par les lecteurs, ce qui témoigne de la compatibilité du texte avec le principe postmoderniste de « l'œuvre ouverte ».

Dans les romans sont réunis des éléments, des conventions, des stratégies propres à différents types de narration ou d'écriture, issus de la littérature de masse et de celle d'élite (le principe « d'attractivité-intellectualité »). En utilisant une histoire d'aventure, Karatkevič éveille la mémoire historique des Biélorusses, il enseigne l'amour de la patrie, de son histoire, de sa langue nationale. Khrystos pryzjamliusja $u$ Garodni est un roman historique d'aventure qui possède certains traits du postmodernisme: «le canvas historique est agrémenté d'éléments d'aventure, les idées de l'auteur se basent sur la culture populaire du rire et les acquis de la littérature mondiale $»^{17}$.

La tentative faite par certains critiques littéraires de retrouver le postmodernisme ex post ,c'est-à-dire dans le passé, a permis de reconnaître Karatkevič comme un précurseur du postmodernisme dans la littérature biélorusse.

Bien que des symptômes du postmodernisme soient détectables déjà dans des textes d'écrivains de la deuxième vague de renaissance nationale, c'est-à-dire du tournant des années 1980 et 1990 (en particulier chez les représentants de «Tutejšyja » et de TVL), la naissance du postmodernisme dans la littérature biélorusse est avant tout associée à l'œuvre d'auteurs de la plus jeune génération, dont celle des membres de la formation littéraire Bum-Bam-Lit, fondée en 1995. En manifestant leurs opinions esthétiques, les jeunes écrivains ont favorisé la diffusion des idées postmodernistes dans leur pays. Au cours des cinq années d'existence de l'association, les représentants de Bum-Bam-Lit ont publié quelques dizaines de volumes poétiques et de recueils de textes de la bibliothèque BBL, ils ont pris part à l'organisation de lectures publiques, de cours de philosophie, de culturologie, d'histoire de l'art et de littérature, de festivals de musique alternative, de littérature et de performances, ils ont entrepris l'édition des revues « NIHIL » et « TEKSTY », ils ont initié la mise en place du projet d'édition underground « DRUGI FRONT MASTACTVA ", dans le cadre duquel sont édités jusqu'à nos jours des textes d'auteurs biélorusses contemporains. Les créations des représentants de Bum-Bam-Lit (Zmicer Višnjou, Illi Sina, Viktar ŽybuL', Serž Minskevič, AL'gerd Bakharevič, Arcjom Kavaleuski, Ganna Cichanova, Ales' Turovič, Usevalad Garačka) sont caractérisées par les expérimentations formelles, la provocation littéraire, la destruction linguistique, la transgression, l'humour noir, la théâtralisation de la littérature, l'introduction de la performance comme genre artistique indépendant. Bum-Bam-Lit a assuré le développement de l'avant-garde biélorusse au cours de la décennie suivante.

Dans les pays d'Europe de l'est, le postmodernisme a remplacé le réalisme socialiste, ce qui a déterminé sa spécificité par comparaison au postmodernisme d'Europe occidentale. Le matériel linguistique du réalisme socialiste et les stéréotypes de la pensée collective 
des hommes soviétiques tout comme la littérature classique des XVIII ${ }^{e}$ et XIX ${ }^{e}$ siècles sont devenus les principaux objets de déconstruction pour les postmodernistes d'Europe de l'est.

Le postmodernisme d'Europe de l'est (en particulier biélorusse) indique une position nonconformiste vis-à-vis du réalisme socialiste « en tant que variété de l'art de masse dans la société totalitaire $»^{18}$. Le traitement postmoderniste «du monde comme un texte» a conditionné le rapport critique à la langue, comprise comme moyen total de manipulation par la conscience des gens. La langue devient le porte-parole de l'idéologie du pouvoir, limitant à cet égard ses possibilités de communication et de connaissance, au prix d'un assortiment donné de constructions linguistiques, d'un lexique schématique, de clichés linguistiques, de slogans pathétiques de propagande, d'invocations vides, de tournures publiques, etc. Les œuvres postmodernistes sont parfois exagérément sociales et politisées, ce qui peut s'expliquer par la situation sociopolitique en Biélorussie. Non seulement les stéréotypes de la pensée soviétique mais aussi les traces visibles du niveau inférieur de la conscience de masse contemporaine, les marques médiocres de l'art officiel, les incompatibilités de la réalité avec les discours des médias, etc. deviennent les objets de négation postmoderniste, laquelle se réalise à l'aide d'une destruction intérieure et d'une reproduction sous une forme grotesque.

Se servant du code double propre à la technique postmoderniste, les représentants du postmodernisme utilisent souvent un lexique licencieux dans leurs œuvres. D'une part, en se basant sur la prise de conscience de la relativité des valeurs morales et esthétiques ainsi que de la pluralité fondamentale de la vérité, les postmodernistes mettent différentes pratiques discursives sur un même pied d'égalité artistique, ils provoquent le lecteur, ils jouent avec ses sentiments esthétiques d'acceptation ou de non acceptation du mot "étranger", grossier ou trop direct. D'autre part, les postmodernistes fixent seulement un état de communication entre les hommes qui repose sur l'utilisation d'un lexique licencieux comme norme non officiellement acceptée des rapports entre les gens. "L'homo sovieticus", le type particulier de l'homme soviétique/postsoviétique, qui réunit un ensemble standard de caractéristiques socio-psychologiques, se trouve au centre de l'attention des postmodernistes: le caractère stéréotypé de la pensée, le schématisme des comportements, l'orientation idéologique, la limitation intellectuelle et le bas niveau de culture. Alors que dans leurs textes les représentants du postmodernisme russe actualisent des éléments anti-esthétiques en vue de discréditer le niveau culturel du peuple, les postmodernistes biélorusses (VoL'ski, Sjargej Mikhalok et de nombreux autres jeunes poètes) se tournent le plus souvent vers une forme de "trasianka $»^{19}$, qui est un mélange de parlers russe et biélorusse, utilisé essentiellement par des gens peu éduqués.

L'art postmoderniste est avant tout un art de reprise qui donne une autre interprétation linguistique et conceptuelle des acquis nationaux des époques culturelles passées. Le fondement premier de la formation du postmodernisme biélorusse ne se trouve ni dans la tradition nationale, ni dans l'européenne. Les raisons tiennent à la spécificité du parcours historique de la littérature biélorusse qui s'est développé non pas en spirale, comme la plupart des littératures mondiales, mais en zigzag, étant donné qu'à une certaine étape on a détruit (et nié) les acquis artistiques et esthétiques passés, et que le développement de la littérature a pris une autre direction. Le réalisme socialiste ne se base pratiquement pas sur la tradition populaire ni sur les acquis polono-biélorusses de la poésie romantique : il a produit son propre héritage. Par conséquent, le lien avec la tradition populaire et les œuvres romantiques biélorusses s'est perdu. Aussi, après la « rupture » 
du réalisme socialiste, la tradition réaliste socialiste a, elle aussi, été réduite à néant. La crise de l'expérience esthétique a été résolue dans le postmodernisme. L'apparition du postmodernisme en Biélorussie (en particulier celui des années 1990) viendrait, semble-til, de la prise de conscience de la nécessité de dépasser le complexe d'infériorité de la littérature nationale, qui est souvent restée hors jeu dans les processus littéraires mondiaux.

Le postmodernisme biélorusse, en se basant sur la tradition européenne et des technologies étrangères, a rencontré une série de difficultés liées à la spécificité de la littérature nationale et à la situation culturelle paradoxale en Biélorussie. La littérature biélorusse ne dispose pas d'un spectre textuel suffisamment large pouvant convenir à la déconstruction ou à l'imitation postmoderniste. Les écrivains biélorusses se tournent souvent vers des œuvres d'art étrangères ou des modèles classiques russes qu'ils adaptent pour leurs compatriotes. Même les stéréotypes de la pensée soviétique sont revus en premier lieu et principalement en russe, et puis ils ont été repris dans des textes biélorusses sous une forme légèrement modifiée. Cela peut s'expliquer par le fait que pendant plusieurs décennies l'art biélorusse est resté dans l'ombre de la littérature russe, se trouvant sous la pression idéologique de la langue d'Aleksandr Puškin, Nikolaj Nekrasov et Lev Tolstoj.

Mais d'un autre côté, le développement du postmodernisme local a été freiné par une connaissance insuffisante de la littérature nationale. Par conséquent, ce n'est pas un hasard si ce sont précisément les classiques biélorusses connus de tous (comme par exemple le poème "A khto tam nja jdze » [Et qui ne vient pas là] de Sidaruk, signé sous le pseudonyme de Nie-Kupala ${ }^{20}$ ), les œuvres ou les aphorismes d'écrivains russes (le récit de Winces' Mudrou « Njačyscik u fraku » [Le diable en frac], dans lequel ont été utilisés des textes de Maksim Gorkij), les mythes héroïques pathétiques de l'époque soviétique, les tournures stéréotypées, les poncifs, la banalité du parler quotidien, etc. qui ont fait l'objet de déconstruction. Hormis cela, le postmodernisme biélorusse a des possibilités limitées $\mathrm{du}$ fait que les œuvres postmodernistes sont avant tout le produit commercial d'une économie de marché développée. Or la littérature biélorusse n'est pas compétitive, même sur le marché national (à la différence de la littérature russe).

39 La maitrise du postmodernisme par les auteurs biélorusses passe le plus souvent par la maitrise des techniques postmodernistes, l'utilisation de procédés, de moyens typiques, d'emprunts artistiques, d'un matériel textuel étranger, etc. En faisant une tentative de classification des figures stylistiques postmodernistes et des moyens artistiques selon leur utilisation fonctionnelle, on peut distinguer trois groupes :

- Le premier est lié à l'intertextualité qui implique l'emploi de citations, la jonction parodique et ironique d'événements et de figures de toutes les époques avec des plans textuels incomparables, les références à l'héritage littéraire, l'assemblage de différents styles et genres artistiques, l'application de la technique du collage et des principes d'intertextualité. Mentionnons à ce titre les poèmes de Gumjanjuk, Sidaruk, Bakharevič, Garačka et Andrej Khadanovič, les récits «Idy na taran!»(Je vais à l'attaque!), " Njačyscik u fraku », « Garačae leta 00-ga » (L'Été très chaud de l'année 00) de Mudrou, la nouvelle Adam Klakocki i jagonyja ceni (Adam Klakocki et son ombre) de Babkou, le texte Imja grušy (Le Nom de la poire) de Sjargej Balakhonau, les romans historiques Litouski Vouk (Le Loup lituanien) d'Ales' Navaryč et Skoki smerci (Les Sauts de la mort) et Zolata zabytykh magilau (L'Or des tombes oubliées) de Ljudmila Rubleuskaja, les romans Bunt nezapratabavanaga prokha (La Révolte des débris inutiles) et Čas zbiracs' kosci : Ewangelle pad 
Luku (Il est temps de rassembler les os : l'Évangile selon Luc) de Viktor Kaz'ko, l'œuvrecitation 1982 d'Aljaksandr Lukašuk, le roman féministe Rekanstrukcyja neba (La Reconstruction du ciel) de Volga Gapeeva, les pièces Stomleny d'jabal (Le Diable fatigué) et Navuka kakhannja (La Science de l'amour) de Sjargej Kavaljov, Vita brevis, abo njagavicy svjatoga Georgija (Vita brevis ou le pantalon de saint Georges) de Miraslau Adamčyk et Črny kvadrat (Le Carré noir) de Klimkovič, Kljatva Gipakrata (Le Serment d'Hippocrate) de VoL'ski, etc.

41 - Le deuxième groupe est celui du jeu, lequel s'accompagne souvent d'une carnavalisation, d'incarnations inattendues, de changements de masques et de rôles, de déplacements temporels et géographiques, d'une diversification de la gamme de couleurs, de l'autodérision du héros lyrique et d'une moquerie de la réalité. Il s'agit par exemple des poèmes de Minskevič, ŻybuL', Džeci ou Vera Burlak, Andrej Bursau, Kavaleuski, Turovič, Vika Trenas, WaLžyna Mort, les textes de Bakharevič et Višnjou.

- Le troisième groupe de procédés postmodernistes comprend le grotesque, la représentation absurde de la réalité, la provocation artistique, l'emploi délibéré d'éléments anti-esthétiques, d'une "poetyka kampu $»^{21}$, d'un lexique licencieux. Citons les œuvres de Globus, notamment Damavikameron, Slavamir Adamovič, Mudrou, Sina, Sidaruk, Gumenjuk, Trenas, Mort, Višnjov, ŽybuL'.

La formation d'un discours féministe ces dernières années dans la littérature biélorusse témoigne aussi de la transition postmoderniste. Les anciennes représentantes de la prose et de la poésie féminine se servaient essentiellement de la langue de l'idéologie masculine dominante dans la culture avec ses valeurs de rationalisme, de force, d'aspiration à un idéal, de lutte pour la primauté et elles apparaissaient dans leurs textes le plus souvent en tant que narratrice sans sexe. L'œuvre des féministes biélorusses de la plus jeune génération (Mort, Gapeev, Džeci ou Burlak, Trenas, Eva Vežnavec) et de la précédente, telles que Svjatlana Aleksievič, Rubleuskaja, Vol'ga Ipatava, est empreinte de révolte, parfois d'agressivité, de rejet de la vision masculine du monde, d'ironie et éventuellement de dérision des stéréotypes masculins de la pensée, de provocation, elle réhabilite la nature et légitime la corporalité comme expérience authentique de la femme, elle oppose l'instinct et la raison, elle reconnaît l'amour comme un moyen de se connaître, appliquant les techniques du kitch, du pastiche, du collage, s'amusant avec les conventions, faisant des jeux de mots, etc.

Le postmodernisme biélorusse contient des réalisations littéraires et artistiques convaincantes, des conceptions philosophiques et théoriques concrètes. Les postmodernistes biélorusses éditent les revues « NIHIL », « PAMIŽ », « PARTISAN », «TEKSTY ». Ils y publient leurs propres œuvres et articles littéraires, critiques, linguistiques, dans lesquels ils s'efforcent de justifier de manière théorique le postmodernisme dans l'art national et international. Un numéro à part de la revue de culturologie «ARCHE $»^{22}$ fut consacré à l'interprétation des problèmes du postmodernisme.

En tout cas, le postmodernisme domine progressivement mais inévitablement le paradigme culturel biélorusse contemporain, il interagit avec d'autres courants et tendances artistiques (le modernisme, le réalisme, l'existentialisme, la néo-avant-garde, l'art de masse), il les modifie et, sous leur influence, il se transforme, s'écartant à certains égards de ses conceptions théoriques et de ses principes esthétiques. 


\section{Conclusion}

46 À la différence du postmodernisme polonais, dont la formation a commencé dans des conditions « underground", soi-disant en opposition avec l'art réaliste socialiste officiel, le postmodernisme biélorusse n'a pas connu de « guérilla » forcée lors de sa formation. Il a remplacé le réalisme socialiste comme une manière artistique alternative d'appréhender le monde, mais pas opposée pour autant. En Pologne, le postmodernisme s'est formé sur un relativement long terme. Il est passé par deux stades : a) les années 1960-1980 (période de formation), b) de la fin des années 1980 à nos jours (période d'authentification et de validation). En Biélorussie, par contre, le postmodernisme s'est manifesté au début des années 1990 et a été confronté à la non-préparation des lecteurs éduqués. Il s'est construit principalement sur les modèles des classiques russes et des œuvres réalistes socialistes.

Le postmodernisme polonais a effectué une réévaluation des idées du modernisme, questionnant souvent son esthétique, alors que l'art postmoderniste russe absorbait les réalisations dissidentes de son propre modernisme, le prenant pour modèle et développant le meilleur de sa tradition. Dans la littérature biélorusse (tout comme dans la littérature ukrainienne), il y a une incompatibilité temporelle signifiante, une absence de lien avec l'art moderniste, qui en son temps ne s'était pas formé comme une orientation complète. C'est pourquoi, à présent, en raison du développement accéléré de la littérature biélorusse, les paradigmes culturels moderniste et postmoderniste se constituent de façon simultanée, s'interpénétrant et interagissant. Ce n'est pas un hasard si des traits du postmodernisme et de l'existentialisme, du « soc-art ${ }^{23}$ et du théâtre de l'absurde, du "schizoréalisme $~^{24}$ et du surréalisme, etc. se retrouvent entremêlés de manière organique dans l'espace d'une seule œuvre.

Chaque peuple possède sa propre voie de développement historico-culturel, qui pourtant n'est pas isolé de la tendance générale de l'évolution de la culture mondiale. C'est pourquoi il ne faut pas étudier la formation de tout phénomène artistique ou esthétique exclusivement dans le cadre de la situation culturelle nationale, mais dans le contexte des autres cultures qui ont témoigné d'une proximité historique, idéologique ou artistique. Par la confrontation et la comparaison de différents discours culturels nationaux, nous pouvons définir les tendances générales du processus littéraire et dégager les propriétés régionales de tel ou tel système artistique de perception du monde.

L'analyse des acquis des littératures polonaise et biélorusse des dernières décennies a donc prouvé qu'un nouveau type de pensée artistique est apparu et que sa formation a été fortement influencée par le postmodernisme, conçu comme un système de points de vue artistiques et scientifiques sur le monde, ce qui a été mis en relief dans la culture et la philosophie occidentales dans la deuxième moitié du XXe siècle. 


\section{NOTES}

1. DĄbrowski M., Postmodernizm : myśl i tekst (Le postmodernisme : pensée et texte), UNIVERSITAS, Cracovie, 2000, p. 25.

2. Ibid., p. 26.

3. Tomkowski J., Dwadzieścia lat z literaturą 1977-1996 (Vingt ans avec la littérature 1977-1996), Państwowy Instytut Wydawniczy, Varsovie, p. 25.

4. DĄbrowski M., Op. cit., p. 25.

5. Suivant de peu la mort de Staline (1953), laquelle a entraîné des changements significatifs en Union soviétique, l'année 1956 est marquée par plusieurs événements clés qui vont mener à un dégel partiel en Pologne. Le premier d'entre eux est le soulèvement de Poznań à la fin du mois de juin, une grève des ouvriers s'étant muée en insurrection générale, sévèrement réprimée par l'armée. Suite à cela, en octobre, l'équipe du PZPR au pouvoir change, avec à sa tête le " modéré " Władysław Gomułka, qui va lancer des réformes démocratiques ( $\mathrm{NdT}$ ).

6. DĄbrowski M., Op. cit., p. 78.

7. Wiegandt E., «Literatura ojczyzn prywatnych a postmodernizm » (La Littérature des patries privées et le postmodernisme), in : Postmodernizm po polsku ? (Le postmodernisme à la polonaise ?) (Sous la direction de Izdebska A. \& Szajnert D.) Wydawnictwo Uniwersytetu Łódzkiego, Łódź, 1998, p. 8.

8. Możejko E., Paradygmaty prozy postmodernistycznej $w$ społeczeństwie totalitarnym na przykładzie literatury polskiej (Les paradigmes de la prose postmoderniste dans la société totalitaire sur l'exemple de la littérature polonaise), in: Postmodernizm $w$ literaturze i kulturze krajów Europy Środkowo-Wschodniej (Le postmodernisme dans la littérature et la culture des pays d'Europe centrale et orientale) (Sous la direction de Fokkema D. W. \& Janaszek-Ivaničková H.), actes du colloque éponyme, 15-19 novembre 1993, Uniwersytet ŚlĄski, Ustroń, Katowice, 1995, p. 90.

9. Uniłowski Krz., Postmodernizm w prozie a debaty krytyczne 1970 - 1987 (Le Postmodernisme en prose et les débats critiques des années 1970-1987), in : Postmodernizm po polsku ?, Op. cit., p. 51.

10. Nowa proza amerykańska (Nouvelle prose américaine), choix de textes établi et introduit par Lewicki Z. (traduits de l'anglais par Anders J., Cendrowska G., Kołyszko A., Lewicki Z. et Wiśniewski J.), Czytelnik, coll. » Nowy Sympozjon », Varsovie, 1983, 434 p.

11. Bolecki W., « Polowanie na postmodernistów (w Polsce) » (La Chasse aux postmodernistes [en Pologne]), in : « TEKSTY DRUGIE », n 1, 1993, pp. 7-24.

12. Łapiński Z., «Postmodernizm - co to i poco ?»(Le postmodernisme : qu'est-ce que c'est et pour quoi ?), in : « TEKSTY DRUGIE », n 1, Op. cit., pp. 74-86.

13. Bartoszyński K., «Postmodernizm a "sprawa polska” - przypadek Miazgi»(Le postmodernisme et la "question polonaise" : le cas de La pulpe), in : « TEKSTY DRUGIE », $\mathrm{n}^{\circ}$ 1, Op. cit., pp. 36-54.

14. Postmodernizm: Encyklopedija (Le postmodernisme: encyclopédie) (Sous la direction de Grycanov A. A. \& Možejko M. A.), Interpresservis, Knižnyj Dom, Minsk, 2001, 1037 p.

15. De son nom latin Matys Strycovius, cet historien, poète, diplomate et prêtre polono-lituanien (1547-1593) écrivait sous le pseudonyme d'Osostevitius. Il est l'auteur de «Kronika Polska, Litewska, Żmudzka i wszystkiej Rusi » (Chronique polonaise, lituanienne, samogitienne et de toute la Rus, 1582).

16. Karatkevič U., « Khrystos pryzjamliusja u Garodni »(Christ a atterri à Grodno), in : Zbor tvorau u 8 t., t. 6, (Sous la direction de Bryl' I. A.), Mastackaja Litaratura, Minsk, 1990, 494 p. 
17. Scjapanava A., Rysy postmadernizmu u tvorčasci Uladzimir Karatkeviča (Les traits du postmodernisme dans l'œuvre de Uladzimir Karatkevič), in : « RODNAE SLOVA », n 4, p. 58.

18. Gumjanjuk J., Postmadernovy praryu... (La percée postmoderne...), in : « KULTURA », n 4, 1995, p. 8.

19. Créole de russe et de biélorusse utilisé en Biélorussie : il s'agit d'une contamination de la phonétique biélorusse dans le lexique russe $(\mathrm{NdT})$.

20. C'est-à-dire « Pas-Kupala ». Il s'agit du poète et dramaturge Janka Kupala (1882-1942). Voir « SLAVICA BRUXELLENSIA », $\mathrm{n}^{\circ}$ 1, Bruxelles, 2008 (NdT).

21. Stylisation qui rend une chose plaisante par son mauvais goût ou son caractère ironique $(\mathrm{NdT})$.

22. « ARCHE », n 1, Minsk, 1999.

23. Tendance dans le courant postmoderniste dans les pays postsoviétiques. Les stéréotypes, les mythes idéologiques, les clichés langagiers de l'époque soviétique y font l'objet d'une déconstruction.

24. Méthode artistique postmoderniste. Ce terme a été introduit dans la critique biélorusse par Jury Basevič.

\section{AUTEURS}

NATALIA DZIENISIUK

Doctorante de l'Institut de langue et de littérature NAN, Mińsk, Biélorussie 